\title{
Evaluación de causas secundarias de baja masa ósea en mujeres colombianas con osteoporosis posmenopáusica
}

\author{
Edwin Antonio Wandurraga Sánchez Md, Msc', Lisseth Fernanda Marín Carrillo Md², Annie \\ Katherine Natera Melo³, Claudia Milena Gómez Giraldo ${ }^{4}$, Juan Camilo Mendoza Díaz
}

\author{
${ }^{1}$ Universidad Autónoma de Bucaramanga, UNAB. Floridablanca, \\ Santander, Colombia. Clínica Carlos Ardila Lulle. Floridablanca, \\ Santander. Colombia. \\ ${ }^{2}$ Hospital Universitario San Ignacio. Unidad de Endocrinología. \\ Bogotá, Colombia. \\ ${ }^{3}$ Hospital Universitario San Ignacio. Unidad de Endocrinología. \\ Bogotá, Colombia. \\ ${ }^{4}$ Hospital Universitario San Ignacio. Unidad de Endocrinología. \\ Bogotá, Colombia. \\ ${ }^{5}$ Universidad Autónoma de Bucaramanga, UNAB. Floridablanca, \\ Santander, Colombia. Clínica Carlos Ardila Lulle. Floridablanca, \\ Santander. Colombia \\ ${ }^{6}$ Universidad Autónoma de Bucaramanga, UNAB. Floridablanca, \\ Santander, Colombia.
}

Datos de correspondencia: Edwin Antonio Wandurraga. Carrera 24 \#154-106, Centro médico Carlos Ardila Lulle, Torre B piso 8 consultorio 806.Floridablanca, Santander. 3133333034. edwinwandurraga@gmail.com

Conflictos de interés

Los autores manifiestan no tener conflictos de interés.

\section{Financiación}

El presente trabajo se realizó con recursos propios. No requirió financiación externa.

Fecha de recepción: 4/09/2016

Fecha de aceptación: 24/10/2016

\section{Resumen}

Introducción: La osteoporosis posmenopáusica puede coexistir con otras entidades que aumentan la pérdida ósea.

objetivo: Determinar la frecuencia de causas secundarias de baja masa ósea en mujeres con osteoporosis posmenopáusica en una población colombiana.

Diseño: Estudio descriptivo retrospectivo.

Población: Mujeres mayores de 50 años con diagnóstico reciente de osteoporosis posmenopáusica antes de iniciar tratamiento.

Mediciones: Se incluyeron variables demográficas, densitométricas y bioquímicas como hemoglobina, fosfatasa alca- lina, transaminasas, creatinina, 25-hidroxivitamina D, calcio, fósforo, magnesio, calciuria en 24 horas, PTH y TSH.

Resultados: Se incluyeron 129 mujeres con edad promedio de $67+/-8,8$ años. Cuarenta y nueve mujeres (36\%) presentaban antecedente de fractura por fragilidad. En el 86,8\% se encontró al menos una alteración bioquímica asociada con pérdida de masa ósea, documentándose insuficiencia de vitamina D en 71,8\%, hiperparatiroidismo normocalcémico en $18,1 \%$ e hipercalciuria en $6,4 \%$. Las mujeres con antecedente de fractura presentaron valor promedio de fosfatasa alcalina superior $(111,6+/-61,3$ vs 87,1 $+/-30,4 \mathrm{U} / \mathrm{L}, p=0,0143$ ) y promedio de hemoglobina inferior $(12,9+/-1,2$ vs. $14,2+/-1,2 \mathrm{gr} / \mathrm{dl}, p<0,0001)$ al compararse con las mujeres sin fractura. Se encontró correlación inversa entre los niveles de fosfatasa alcalina y la densidad mineral ósea de la columna lumbar $(p<0,001)$ y la cadera $(p=0,003)$.

Conclusiones: Las causas secundarias de baja masa ósea en mujeres con OPM son frecuentes en nuestro medio. Con base en una frecuencia de alteraciones mayor al 5\%, sugerimos la evaluación de toda mujer con OPM con hemoglobina, calcio, calciuria en 24 horas, 25-hidroxivitamina D, AST, PTH y TSH.

Palabras clave: Osteoporosis posmenopáusica; fractura osteoporótica; etiología; densidad mineral ósea; deficiencia de vitamina D; hiperparatirodismo secundario; hipercalciuria.

\section{Abstract}

Introduction: Postmenopausal osteoporosis can coexist with other entities that increase bone loss.

Aim: To determine the frequency of secondary causes of low bone mass in women with postmenopausal osteoporosis in a Colombian population.

Materials and methods: $A$ retrospective descriptive study was conducted, including women over 50 years with newly diagnosed postmenopausal osteoporosis without treatment. Demographic, densitometric and biochemical variables such as hemoglobin, alkaline phosphatase, transaminases, creatinine, 25 hydroxivitamin D, calcium, phosphorus, magnesium, calciuria in 24 hours, PTH and TSH were evaluated. 
Results: 129 women with a mean age of $67+/-8,8$ years were included. 49 patients (36\%) had history of fragility fracture. At least one biochemical disorder associated with bone loss was reported in $86,8 \%$ of cases, vitamin D insufficiency was documented in $71,8 \%$, normocalcemic hyperparathyroidism in $18,1 \%$ and hypercalciuria in $6,4 \%$. Women with history of fracture showed higher average value of alkaline phosphatase $(111,6+/-61,3 \mathrm{vs}$ $87,1+/-30,4 U / L, p=0,0143)$ and lower mean hemoglobin $(12,9$ $+/-1,2$ vs 14,2 +/- 1,2 gr/dl, $p<0,0001)$ compared with women without fracture. Inverse correlation was found between levels of alkaline phosphatase and bone mineral density of lumbar spine $(p<0,001)$ and hip $(p=0,003)$.

Conclusions: Secondary causes of low bone mass in women with PMO are frequent in our clinical practice. Based on a frequency of laboratory abnormalities greater than 5\%, we suggest that all women with PMO should be studied with hemoglobin, serum calcium, urinary calcium in 24 hours, 25 hydroxivitamin D, AST, PTH and TSH.

Keywords: Postmenopausal osteoporosis; osteoporotic fracture; etiology; bone mineral density; vitamin D deficiency; secondary hyperparathyroidism; hypercalciuria.

\section{Introducción}

La osteoporosis posmenopáusica (OPM) es la enfermedad ósea metabólica más frecuente, caracterizada por baja densidad mineral ósea y deterioro de la microarquitectura con incremento en la fragilidad ósea y mayor riesgo de fractura $^{(1)}$. Se trata de un trastorno presente hasta en el $20 \%$ de las mujeres posmenopáusicas, y aproximadamente un 50\% desarrollará fracturas por fragilidad durante el resto de su vida ${ }^{(1-3)}$. Además de la deprivación estrogénica y el riesgo propio de la edad, existen otras causas de pérdida de masa ósea en mujeres con OPM que se deben tener en cuenta como: deficiencia de vitamina D, trastornos tiroideos, hiperparatiroidismo, hipercortisolismo, diabetes mellitus, hipercalciuria idiopática, hepatopatía crónica, enfermedad celiaca, cirugía bariátrica, hipomagnesemia, trastornos hematológicos como el mieloma múltiple y fármacos (anticonvulsivantes, ciclosporina, glucocorticoides, agonistas de la hormona liberadora de gonadotropina, inhibidores de la aromatasa e inhibidores de bomba de protones), entre otros ${ }^{(4-7)}$. Hasta en el $30 \%$ de mujeres posmenopáusicas se han reportado causas secundarias de osteoporosis, que se asocian significativamente a alteraciones como hipercalciuria, deficiencia de vitamina $D$ e hiperparatiroidismo, mientras que en hombres este porcentaje alcanza del $50 \%$ al $80 \%{ }^{(4-9)}$. Para el diagnóstico de causas secundarias se recomienda realizar exámenes de laboratorio de rutina que incluyan hemoglobina, calcio, fósforo, pruebas de función hepática, perfil tiroideo, función renal, 25-hidroxivitamina D, PTH y calcio en orina de 24 horas y, en casos especiales, pruebas de laboratorio especializado dirigidas $^{(2,3)}$. La importancia de documentar causas secun- darias radica en la pobre respuesta que puede obtenerse con la terapia específica para la OPM cuando el desorden no se reconoce $^{(7)}$. Teniendo en cuenta el costo alto que implica la realización de múltiples laboratorios de rutina, fue nuestra intención determinar la prevalencia de causas secundarias de baja masa ósea en mujeres con osteoporosis posmenopáusica atendidas por endocrinología en dos centros de Colombia y, con base en los resultados, proponer un paquete básico de paraclínicos que se deben realizar de forma sistemática en esta población.

\section{Materiales y métodos}

Se realizó un estudio descriptivo retrospectivo de corte transversal en donde se incluyeron mujeres mayores de 50 años con diagnóstico reciente de OPM sin causa evidente secundaria, valoradas en la consulta externa de los servicios de endocrinología de dos ciudades en Colombia (Bogotá y Bucaramanga) en un periodo de tres años. Se definió osteoporosis según criterio densitométrico con valores de $\mathrm{T}$ en columna vertebral, fémur o radio distal igual o menor a $-2,5 \mathrm{DE}$ o por la presencia de un valor de $\mathrm{T}<-1,0 \mathrm{DE}$ con una o más fracturas por fragilidad. Posmenopausia se definió como más de dos años de ausencia de menstruación. Se excluyeron pacientes que se encontraran con manejo farmacológico establecido para la osteoporosis. El estudio fue aprobado por el comité de ética de una de las instituciones participantes.

\section{Variables evaluadas}

La información recolectada se registró en una base de datos que contenía variables demográficas, clínicas y paraclínicas, incluyendo edad, presencia de comorbilidades, historia personal o familiar de fracturas asociada a trauma de bajo impacto, densidad mineral ósea $\left(\mathrm{g} / \mathrm{cm}^{2}\right)$, Valor de $\mathrm{T}$ y $\mathrm{Z}$ de columna lumbar, cadera y/o radio 33\% en desviaciones estándar tomados de la densitometría ósea al momento del diagnóstico, niveles de hemoglobina $(\mathrm{Hb})(\mathrm{g} / \mathrm{dl})$, fosfatasa alcalina $(\mathrm{U} / \mathrm{L})$, transaminasas (U/L), 25-hidroxivitamina D $(\mathrm{ng} / \mathrm{ml})$, calcio sérico $(\mathrm{mg} / \mathrm{dl})$, fósforo sérico $(\mathrm{mg} / \mathrm{dl})$, magnesio sérico $(\mathrm{mg} /$ $\mathrm{dl}$ ), calcio en orina de 24 horas ( $\mathrm{mg} / 24$ horas), PTH (pg/ml) y TSH (mlU/L). Las densitometrías óseas fueron realizadas en centros de referencia avalados en las ciudades participantes, utilizando equipos LUNAR- GE y HOLOGIC.

Se consideró anemia un nivel de $\mathrm{Hb}$ menor a $12 \mathrm{~g} / \mathrm{dl}$, hipocalciuria niveles calcio en orina de 24 horas menores a 100 $\mathrm{mg} / 24$ horas e hipercalciuria mayor a $250 \mathrm{mg} / 24$ horas. Se definió insuficiencia y deficiencia de vitamina $\mathrm{D}$ con valores inferiores a $30 \mathrm{ng} / \mathrm{ml}$ y $20 \mathrm{ng} / \mathrm{ml}$, respectivamente. Los rangos de referencia para las demás pruebas bioquímicas y hormonales fueron determinados por los insertos de cada prueba.

\section{Análisis estadístico}

La muestra se describió empleando medias y desviaciones estándar para variables continuas. Para el caso de las varia- 
bles categorizadas de acuerdo con los puntos de corte de los valores de exámenes de laboratorio se calcularon proporciones con su respectivo IC 95\%, según el antecedente de fractura. La diferencia entre las medias de las variables independientes (variables paraclínicas) en las categorías de historia de fractura, se evaluó con la prueba T de Student. Para valorar la relación entre la edad y los exámenes de laboratorio, se proyectaron diagramas de dispersión y se estimaron modelos de regresión lineal para obtener el coeficiente y el valor p. El análisis se realizó con el paquete estadístico STATA/IC 11,2. Se determinó un nivel de significancia de 5\% bilateral.

\section{Resultados}

Se incluyeron 129 mujeres posmenopáusicas con una edad promedio de $67+/-8,8$ años de las cuales 49 (37,9\%) presentaban antecedente de fractura asociada a trauma de bajo impacto. En la tabla 1 se describen las características de la población. En el 86,8\% de las mujeres se encontró al menos una alteración adicional relacionada con pérdida de masa ósea. Se documentó insuficiencia de vitamina D en el 71,8\% de la población, seguido por hiperparatiroidismo normocalcémico en el $18,1 \%$ e hipercalciuria en el 6,4 \%. La frecuencia de las alteraciones metabólicas encontradas en la población puede observarse en la tabla 2 . Al analizar los valores de laboratorio de las pacientes, según su historia de fractura, se encontró un valor promedio de fosfatasa alcalina en las mujeres fracturadas de 111,6 U/L+/-61,3 U/L y de 87,1 U/l+/-30,4 U/L en las mujeres sin historia de fractura $(p=0,0143)$. El promedio de hemoglobina en las mujeres con historia de fractura fue de $12,9 \mathrm{~g} / \mathrm{dl}(+/-1,2 \mathrm{~g} / \mathrm{dl})$ versus $14,2 \mathrm{~g} / \mathrm{dl}(+/-1,2)$ sin este antecedente $(p<0,0001)$. No se encontraron otras diferencias en los demás valores de laboratorio, según el antecedente de fractura (tabla 3). Cuando se analizó la relación entre los resultados de las pruebas bioquímicas con la densidad mineral ósea (DMO), se encontró una correlación inversa entre los niveles de fosfatasa alcalina y la DMO a nivel de columna lumbar (coef: $-183,5$ UI/L por $\mathrm{g} / \mathrm{cm}^{2}, p<0,001$ ) y de cadera (coef: $-122,6 \mathrm{UI} / \mathrm{L}$ por $\mathrm{g} / \mathrm{cm}^{2}, p=0,003$. No se encontró correlación entre las demás variables bioquímicas y la densidad mineral ósea. En cuanto a la distribución de las alteraciones metabólicas con la edad se encontró una correlación inversa entre la edad y los niveles de calciuria en 24 horas (correlación: $-0,25, p=0,009$ y entre la edad y la DMO en cadera (correlación: - $0,42, p=0,000$ ).

\section{Discusión}

Nuestro estudio documentó una alta frecuencia de desórdenes metabólicos asociados a baja masa ósea en mujeres con OPM, lo que refuerza la idea del tamizaje de rutina. El 71,8\% de las pacientes evaluadas tenían niveles de 25 hidroxivitamina D3 menores a $30 \mathrm{ng} / \mathrm{ml}$. Esta frecuencia es similar a la encontrada por Cerdá y cols. (82\%) y por Quesada-Gómez y cols. $(76,4 \%)$
Tabla 1. Características demográficas y clínicas de la población estudiada (129 mujeres con osteoporosis posmenopáusica)

\begin{tabular}{|c|c|}
\hline Característica & n (\%) \\
\hline Edad (años) & $67+/-8,8 *$ \\
\hline \multicolumn{2}{|l|}{ Ciudad de origen } \\
\hline Bogotá & $76(58,9 \%)$ \\
\hline Bucaramanga & $53(41,1 \%)$ \\
\hline \multicolumn{2}{|l|}{ Comorbilidad } \\
\hline Hipertensión arterial & $40(31 \%)$ \\
\hline Diabetes mellitus & $31(24 \%)$ \\
\hline Cáncer & $25(19,3 \%)$ \\
\hline Mama & $11(8,5 \%)$ \\
\hline Tiroides & $5(3,8 \%)$ \\
\hline Hematolinfoide & $3(2,3 \%)$ \\
\hline Cervix & $3(2,3 \%)$ \\
\hline Otros & $3(2,3 \%)$ \\
\hline Hipotiroidismo & $11(8,5 \%)$ \\
\hline Insuficiencia cardiaca & $10(7,7 \%)$ \\
\hline Enfermedad coronaria & $8(6,2 \%)$ \\
\hline $\begin{array}{l}\text { Enfermedad pulmonar obstructiva } \\
\text { crónica }\end{array}$ & $8(6,2 \%)$ \\
\hline \multicolumn{2}{|l|}{ Densidad mineral ósea $\left(\mathrm{g} / \mathrm{cm}^{2}\right)$} \\
\hline Columna lumbar $(n=122)$ & $0,819+/-0,112 *$ \\
\hline Cuello de fémur (n=122) & $0,729+/-0,108 *$ \\
\hline Radio $33 \%(n=22)$ & $0,591+/-0,131 *$ \\
\hline \multicolumn{2}{|l|}{ Valor de T (desviación estándar) } \\
\hline Columna lumbar & $-2,86+/-0,87 *$ \\
\hline Cuello de fémur & $-2,14+/-0,80 *$ \\
\hline Radio 33\% & $-3,40+/-1,13 *$ \\
\hline \multicolumn{2}{|l|}{ Valor de Z (desviación estándar) } \\
\hline Columna lumbar & $-1,38+/-0,84 *$ \\
\hline Cuello de fémur & $-0,55+/-0,72 *$ \\
\hline Radio 33\% & $-1,30+/-0,99 *$ \\
\hline Antecedente de fractura & $49(37,9 \%)$ \\
\hline Columna vertebral & $22(17 \%)$ \\
\hline Antebrazo & $14(10,8 \%)$ \\
\hline Cadera & $7(5,4 \%)$ \\
\hline
\end{tabular}

*Promedio con desviación estándar

en poblaciones de España ${ }^{(8,9)}$. De igual manera, se encontró en nuestra serie, deficiencia de vitamina $\mathrm{D}(<20 \mathrm{ng} / \mathrm{ml})$ en el $22,7 \%$, cifra muy parecida a la reportada en el trabajo de Tannenbaum y cols. ${ }^{(10)}$ en un centro de referencia en Nueva York (21,3\%). Estas similitudes resultan llamativas para nuestro país, ya que nos encontramos en una región del trópico carente de estaciones y con mayor exposición a rayos ultravioleta, desmitificando la creencia de que no tenemos deficiencia de vitamina D por nuestra ubicación geográfica. Este hallazgo valida lo descrito previamente en Colombia ${ }^{(11,12)}$ y en otros países tropicales como Brasil ${ }^{(13)}$ con una prevalencia de deficiencia de 
Tabla 2. Frecuencia de las alteraciones bioquímicas en 129 mujeres con osteoporosis posmenopáusica

\begin{tabular}{|c|c|c|c|}
\hline Alteración de laboratorio & $\begin{array}{c}\text { n } \\
\text { Diagnóstico }\end{array}$ & $\begin{array}{c}\text { n } \\
\text { Con dato de la } \\
\text { variable de interés }\end{array}$ & $\begin{array}{c}\% \\
\text { Sujetos con la } \\
\text { alteración }\end{array}$ \\
\hline Vitamina $\mathrm{D}(<30 \mathrm{ng} / \mathrm{ml})$ & 79 & 110 & 71,8 \\
\hline Vitamina $\mathrm{D}(<20 \mathrm{ng} / \mathrm{ml})$ & 25 & 110 & 22,7 \\
\hline $\begin{array}{l}\text { Calcio en orina de } 24 \text { horas disminuido } \\
(<100 \mathrm{mg} / 24 \text { horas })\end{array}$ & 54 & 109 & 49,5 \\
\hline $\begin{array}{l}\text { Calcio en orina de } 24 \text { horas elevado } \\
\text { (> } 250 \mathrm{mg} / 24 \mathrm{hrs})\end{array}$ & 7 & 109 & 6,4 \\
\hline PTH elevada (> 65 pg/ml) & 21 & 116 & 18,1 \\
\hline Fosfatasa alcalina elevada * & 15 & 88 & 17 \\
\hline Anemia $(\mathrm{Hb}<12 \mathrm{gr} / \mathrm{dl})$ & 9 & 101 & 8,9 \\
\hline TSH baja * & 9 & 120 & 7,5 \\
\hline AST elevada * & 3 & 52 & 5,7 \\
\hline ALT elevada * & 2 & 52 & 3,8 \\
\hline Hipocalcemia * & 10 & 123 & 8,1 \\
\hline Hipofosfatemia * & 2 & 95 & 2,1 \\
\hline Hipomagnesemia * & 1 & 40 & 2,5 \\
\hline Creatinina elevada * & 2 & 118 & 1,7 \\
\hline
\end{tabular}

*Valor superior al límite alto del laboratorio de referencia

Tabla 3. Comparación de los valores de las pruebas de laboratorio según el antecedente de fractura por fragilidad

\begin{tabular}{|c|c|c|c|c|c|c|c|}
\hline \multirow[b]{2}{*}{ Prueba de laboratorio } & \multicolumn{3}{|c|}{ Historia de fractura por fragilidad } & \multicolumn{3}{|c|}{ Sin historia de fractura por fragilidad } & \multirow[b]{2}{*}{ Valor de $\mathrm{p}$} \\
\hline & $\mathbf{n}$ & Promedio & DE & $\mathbf{n}$ & Promedio & DE & \\
\hline $25 \mathrm{OH}$ vitamina $\mathrm{D}(\mathrm{ng} / \mathrm{ml})$ & 42 & 26,4 & 9,3 & 67 & 26,4 & 10,0 & 0,9741 \\
\hline Calciuria (mg/24 horas) & 41 & 120,1 & 86,1 & 68 & 124,5 & 75,2 & 0,7758 \\
\hline PTH (pg/ml) & 44 & 49,9 & 28,7 & 72 & 55,8 & 28,9 & 0,2868 \\
\hline Fosfatasa alcalina (UI/L) & 33 & 111,6 & 61,3 & 55 & 87,1 & 30,4 & $0,0143^{*}$ \\
\hline Hemoglobina (gr/dl) & 33 & 12,9 & 1,2 & 57 & 14,2 & 1,2 & $<0,0001^{*}$ \\
\hline TSH (mUI/L) & 44 & 2,8 & 3,1 & 75 & 3,1 & 2,9 & 0,5837 \\
\hline AST (UI/L) & 20 & 22,8 & 4,9 & 32 & 24,5 & 10,0 & 0,4633 \\
\hline ALT (UI/L) & 20 & 23,1 & 8,2 & 32 & 23,6 & 10,4 & 0,8431 \\
\hline Calcemia (mg/dl) & 48 & 9,3 & 0,9 & 76 & 9,3 & 0,7 & 0,8318 \\
\hline Fosfatemia (mg/dl) & 32 & 3,7 & 0,7 & 67 & 3,7 & 0,4 & 0,7811 \\
\hline Creatinina (mg/dl) & 42 & 0,8 & 0,1 & 75 & 0,8 & 0,2 & 0,3521 \\
\hline
\end{tabular}

*Valor de $p$ con significancia estadística menor a 0,05

vitamina D del 28,4\%, en Camerún ${ }^{(14)}$ del $24 \%$ y en Indonesia ${ }^{(15)}$ del $35,1 \%$, contrastando con países como Ghana y Jamaica con una prevalencia del 4,6\% y 8,3\%(16). Hábitos de vestimenta, costumbres de exposición solar, el uso de antisolares y el tipo de dieta podrían explicar estas diferencias regionales ${ }^{(17,18)}$. Una de cada cinco pacientes en nuestra serie presentaba hiperparatiroidismo normocalcémico. Dado que la enfermedad renal crónica fue rara, se consideró eran secundarios a deficiencia de vitamina $\mathrm{D}$, sin poder descartar un hiperparatiroidismo primario incipiente. Esta cifra fue similar a la reportada en Norteamérica $(15,6 \%)$ e inferior a la de España $(35 \%)^{(8,10)}$. De igual manera, encontramos una frecuencia de hipercalciuria similar a la serie americana $(6,4 \%$ y $9,8 \%$ respectivamente) e inferior a la española, en donde se reportó en el $20 \%$ de las mujeres eva- 
luadas $^{(8,10)}$. Es importante este hallazgo, ya que la identificación de la hipercalciuria idiopática en nuestras pacientes sería susceptible de intervención con una dieta normal en calcio y con el uso de diuréticos tipo tiazidas ${ }^{(19)}$. Por otro lado, encontramos una tasa alta de hipocalciuria, siendo del $49,5 \%$ y del $18,3 \%$ con calcio $<100 \mathrm{mg} / 24$ horas y $<50 \mathrm{mg} / 24$ horas, respectivamente. Como posibles causas consideramos el consumo dietario bajo de calcio en Colombia, tal como se registró en la encuesta nacional de la situación nutricional (ENSIN 2005) en donde el 85,8\% de las personas no cubría los requerimientos mínimos ${ }^{(20)}$, y la alta prevalencia de insuficiencia de vitamina D que limitaría la biodisponibilidad del calcio de la dieta por disminución en la absorción intestinal. Como hallazgo adicional, se registró menor calciuria a mayor edad (coef $-2,2, \mathrm{p}=0,009$ ); es decir, por cada año cumplido se disminuía en $2,2 \mathrm{mg}$ el calcio en orina. No se encontró relación entre la calciuria y la DMO en columna o en cadera ni con la presencia de fractura. Documentamos una relación inversa entre los niveles de fosfatasa alcalina con la DMO de columna y cadera. Así mismo, el nivel promedio de esta enzima fue mayor en las mujeres con antecedente de fractura, reflejando un recambio óseo acelerado ${ }^{(21,22)}$, por lo que su papel potencial como marcador de fractura o en el seguimiento de las terapias para OPM debe ser investigado. Cabe mencionar que no se encontraron casos de enfermedad de Paget del hueso, metástasis óseas o hepatopatía significativa. Por último, se reportó un menor nivel de $\mathrm{Hb}$ en las pacientes con historia de fractura.
Respecto a esto, Korkmaz y cols. en Turquía descubrieron valores más bajos de DMO a nivel de fémur y de columna vertebral en mujeres con OPM y anemia respecto a mujeres con $\mathrm{Hb}$ nor$\mathrm{mal}^{(23)}$. Como hipótesis de la mayor prevalencia de osteoporosis en pacientes con anemia, independiente de su etiología, se plantea que la pérdida de sangre continua estimula la proliferación de células hematopoiéticas, incluyendo a los osteoclastos y de células progenitoras estromales que originan a los osteoblastos, responsables de la mayor tasa de recambio óseo ${ }^{(24)}$. Adicionalmente, se ha descrito mayor número de caídas, mareo crónico e hipotensión ortostática en pacientes con anemia ${ }^{(25,26)}$.

\section{Conclusión}

La prevalencia de causas secundarias de baja masa ósea en mujeres con OPM, tal como ha sido descrito en otras poblaciones, resulta frecuente en nuestro medio. Con base en una frecuencia de trastornos metabólicos mayor al 5\%, sugerimos la evaluación de toda mujer con osteoporosis posmenopáusica con hemoglobina, calcio sérico, calcio en orina 24 horas, 25 hidroxivitamina D, AST, PTH y TSH.

\section{Agradecimientos}

Agradecemos a Johanna Otero Wandurraga por su asesoría en epidemiología, y a Pedro Iván Pinto y Javier Noriega Rangel por la ayuda en la recolección de información.

\section{Referencias}

1. Nochowitz B, Siegert S and Wasik M. An Update on Osteoporosis. Am J Ther. 2009 Sep-Oct;16(5):437-45.

2. Kanis JA, McCloskey EV, Johansson H, Cooper C, Rizzoli R, Reginster JY, et al. European guidance for the diagnosis and management of osteoporosis in postmenopausal women. Osteoporos Int. 2013 Jan;24(1):23-57.

3. Cosman F, de Beur SJ, Leboff MS, Lewiecki EM, Tanner B, Randall S, et al. Clinician's Guide to prevention and treatment of Osteoporosis. Osteoporos Int. 2014 Oct;25(10):2359-81.

4. Armas LA, Recker RR. Pathophysiology of osteoporosis: new mechanistic insights. Endocrinol Metab Clin North Am. 2012 Sep;41(3):475-86.

5. Mirza F, Canalis E. Management of endocrine disease: secondary osteoporosis: pathophysiology and management. Eur J Endocrinol. 2015 Sep;173(3):R131-51.

6. Stein E, Shane E. Secondary osteoporosis. Endocrinol Metab Clin N Am 2003;32:115-134.

7. Kelman A, Lane NE. The management of secondary osteoporosis. Best Pract Res Clin Rheumatol 2005 Dec;19(6):1021-37.

8. Cerdá D, Peris P, Monegal A, Albaladejo C, Martínez MA, Muxí A, et al. Search for hidden secondary causes in postmenopausal women with osteoporosis. Menopause 2010 Jan-Feb;17(1):135-9.

9. Quesada-Gómez JM, Diaz-Curiel M, Sosa-Henriquez M, Malouf-Sierra J, Nogues-Solan X, Gomez-Alonso C, et al. Low calcium intake and inadequate vitamin D status in postmenopausal osteoporotic women. J Steroid Biochem Mol Biol. 2013 Jul;136:175-7.

10. Tannenbaum C, Clark J, Schwartzman K, Wallenstein S, Lapinski R, Meier D, et al. Yield of Laboratory Testing to Identify Secondary Contributors to Osteoporosis in Otherwise Healthy Women. J Clin Endocrinol Metab 2002 Oct;87(10):4431-7.

11. Molina JF, Molina J, Escobar JA, Betancur JF, Giraldo A. Niveles de 25 hidroxivitamina D y su correlación clínica con diferentes variables metabólicas y cardiovasculares en una población de mujeres posmenopáusicas Acta MedColomb vol. 36 no. 1 Bogotá Jan./Mar.2011.

12. Gonzalez D, Zuñiga C, K Kattah W. Insuficiencia de vitamina D en pacientes adultos con baja masa ósea y osteoporosis en la Fundación Santa Fe de Bogotá 2008-2009. Rev Col Reumatol 2010 17(4):212-218.

13. Correia A, Azevedo Mdo S, Gondim F, Bandeira F. Ethnic aspects of vitamin D deficiency. Arq Bras Endocrinol Metabol. 2014 Jul;58(5):540-4.

14. Njemini R, Meyers I, Demanet C, Smitz J, Sosso M, Mets T. The prevalence of antibodies in an elderly sub-Saharan African population. Clin Exp Immunol 2002 Jan;127(1):99-106.

15. Setiati S. Vitamin D status among Indonesian eldery women living in institutionalized care units. Acta Med Indones. $2008 \mathrm{Apr} ; 40(2): 78-83$.

16. Durazo-Arvizu RA, Camacho P, Bovet P, Forrester T, Lambert EV, PlangeRhule J, et al. 25-Hydroxyvitamin D in African-origin populations at varying latitudes challenges the construct of a physiologic norm. Am J Clin Nutr. 2014 Sep;100(3):908-14.

17. Van Schoor NM, Lips P. Worldwide vitamin D status. Best Pract Res Clin Endocrinol Metab 2011 Aug;25(4):671-80.

18. Arantes HP, Kulak CA, Fernandes CE, Zerbini C, Bandeira F, Barbosa IC, et al Correlation between 25-hydroxyvitamin D levels and latitude in Brazilian postmenopausal women: from the Arzoxifene Generations Trial. Osteoporos Int. 2013 Oct;24(10):2707-12.

19. Escribano I, Balaguer A, Pagone F, Feliu A, Roqué I Figuls M. Pharmacological interventions for preventing complications in idiopathic hypercalciuria. Cochrane Database Syst Rev. 2009 Jan 21;(1):CD004754.

20. Ministerio de salud y protección social. Encuesta nacional de la situación nutricional en Colombia, 2005. Fecha de consulta: 30 de agosto de 2016 www.minsalud.gov.co/sites/rid/Lists/BibliotecaDigital/RIDE/VS/ED/GCFI/ Ensin\%202005.pdf.

21. Mukaiyama K, Kamimura M, Uchiyama S, Ikegamin S, Nakamura Y, Kato H. Elevation of serum alkaline phosphatase (ALP) level in postmenopausal women is caused by high bone turnover. Aging Clin Exp Res.2015 Aug;27(4):413-8.

22. Atalay S, Elci A, Kayadibi H, Onder CB, Aka N. Diagnostic utility of osteocalcin, undercarboxylated osteocalcin, and alkaline phosphatase for osteoporosis in premenopausal and postmenopausal women. Ann Lab Med. 2012 Jan;32(1):23-30.

23. Korkmaz U, Korkmaz N, Yazici S, Erkan M, Baki AE, Yazici, et al. Anemia as a risk factor for low bone mineral density in postmenopausal Turkish women. Eur J Intern Med. 2012 Mar;23(2):154-8. http://www.ncbi.nlm.nih.gov/ pubmed/22284246

24. Gurevitch O, Slavin S. The hematological etiology of osteoporosis. Med hypotheses. 2006;67(4):729-35

25. Duh MS, Mody SH, Lefebvre P, Woodman RC, Buteau S, Piech CT. Anaemia and the risk of injurious falls in a community-dwelling elderly population. Drugs Aging 2008;25(4):325-34.

26. Penninx BW, Pluijm SM, Lips P, Woodman R, Miedema K, Guralnik JM, et al. Late- life anemia is associated with increased risk of recurrent falls. J Am Geriatr Soc. 2005 Dec;53(12):2106-11. 